\title{
A Rare Form of Pica
}

\author{
Daniel Kurtz ${ }^{1}$, Ibrahim Azar², and Indryas Woldie ${ }^{2}$ \\ ${ }^{1}$ Detroit Medical Center \\ ${ }^{2}$ Karmanos Cancer Center
}

May 5, 2020

\begin{abstract}
Here we present a case report of Xylophagia
\end{abstract}

\section{A Rare Form of Pica}

Daniel Kurtz, $\mathrm{MD}^{1}$, Ibrahim Azar, $\mathrm{MD}^{2}$, Indryas Woldie, $\mathrm{MD}^{2}$

Affiliations:

1 - Department of Internal Medicine, Wayne State University School of Medicine and Detroit Medical Center, Detroit, MI, USA

2 - Department of Oncology, Wayne State University, Karmanos Cancer Center, Detroit, MI, USA

Disclosure: We have no conflicts of interest to declare

Corresponding author: Daniel Kurtz M.D.

Corresponding author contact information: fs9227@wayne.edu

Author contribution statement: Daniel Kurtz and Ibrahim Azar wrote the manuscript. All authors reviewed the final manuscript.

A 32-year-old woman with a history of homozygous sickle cell anemia (Hgb SS disease) was started on anticoagulation with apixaban 5mg BID for superior vena cava thrombosis and pulmonary embolism. She developed hematochezia on anticoagulation and workup revealed iron deficiency anemia [ferritin $8.1 \mathrm{ng} / \mathrm{ml}$ (11-306.8 ng/ml), Hemoglobin 7.0gm/dl (11.5-15.1 gm/dl), MCV $77.4 \mathrm{fl}(82-97 \mathrm{fl}]$. Colonoscopy revealed extensive foreign body, throughout the colon making it difficult to advance the colonoscope beyond the sigmoid colon. Patient was given additional polyethylene glycol 3350 and electrolytes followed by repeat colonoscopy with assistance from large volume of water evacuation, which showed small fragments of toilet paper (Figure A, B, arrows ) up to the terminal ileum. No active source of bleeding was identified. Upon further questioning, patient reported pica and craving for toilet paper and endorsed eating up to two rolls of toilet paper daily. She received three doses of intravenous iron replacement therapy with resolution of her pica and normalization of her ferritin. Xylophagia is a form of pica where patients have the unusual craving for ingestion of paper [1].

Key Clinical Message: Xylophagia is a form of pica where patients have the unusual craving for ingestion of paper. Cravings can resolve after iron replacement therapy.

References: 
Coltman CA. Pagophagia and iron lack. JAMA. 2004;207:513-6
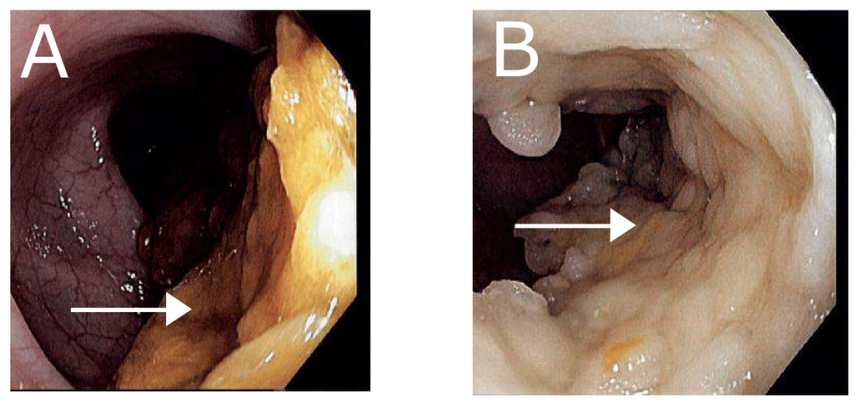